\title{
A Review of Resting-State Electroencephalography Analysis in Disorders of Consciousness
}

\author{
Yang Bai', Xiaoyu Xia ${ }^{2}$ and Xiaoli $\mathrm{Li}^{3 *}$ \\ 1 Institute of Electrical Engineering, Yanshan University, Qinhuangdao, China, ${ }^{2}$ Department of Neurosurgery, PLA Army \\ General Hospital, Beijing, China, ${ }^{3}$ State Key Laboratory of Cognitive Neuroscience and Learning, IDG/McGovern Institute for \\ Brain Research, Beijing Normal University, Beijing, China
}

Recently, neuroimaging technologies have been developed as important methods for assessing the brain condition of patients with disorders of consciousness (DOC). Among these technologies, resting-state electroencephalography (EEG) recording and analysis has been widely applied by clinicians due to its relatively low cost and convenience. EEG reflects the electrical activity of the underlying neurons, and it contains information regarding neuronal population oscillations, the information flow pathway, and neural activity networks. Some features derived from EEG signal processing methods have been proposed to describe the electrical features of the brain with DOC. The computation of these features is challenging for clinicians working to comprehend the

\section{OPEN ACCESS}

Edited by:

Olivia Gosseries,

University of Liège, Belgium

Reviewed by:

Quentin Noirhomme,

Maastricht University, Netherlands

Damian Cruse,

University of Western Ontario,

Canada

*Correspondence:

Xiaoli Li

xiaoli@bnu.edu.cn

Specialty section:

This article was submitted

to Applied Neuroimaging,

a section of the journal

Frontiers in Neurology

Received: 25 June 2017

Accepted: 25 August 2017

Published: 11 September 2017

Citation:

Bai Y, Xia X and Li X (2017)

A Review of Resting-State

Electroencephalography Analysis in Disorders of Consciousness.

Front. Neurol. 8:471.

doi: 10.3389/fneur.2017.00471 corresponding physiological meanings and then to put them into clinical applications. This paper reviews studies that analyze spontaneous EEG of DOC, with the purpose of diagnosis, prognosis, and evaluation of brain interventions. It is expected that this review will promote our understanding of the EEG characteristics in DOC.

Keywords: electroencephalography, disorder of consciousness, vegetative state, minimally conscious state, unresponsive wakefulness syndrome

\section{INTRODUCTION}

Following severe damage to the brain, caused by trauma, stroke, or anoxia, patients may fall into a coma $(1,2)$. When they move out of a coma, they may evolve into a vegetative state (VS) or a minimally conscious state (MCS) according to observable behavioral features (3). Among them, VS (4), or unresponsive wakefulness syndrome (5), is defined by periods of preserved behavioral arousal (6), but unresponsiveness to external stimuli and an absence of awareness (7). MCS shows signs of fluctuating yet reproducible remnants of non-reflex behaviors (8). The disorders of consciousness (DOC) including coma, VS, and MCS pose challenges to clinicians and neuroscientists for diagnosis, treatment, and daily care $(3,9,10)$. A correct diagnosis of MCS and VS is of decisive importance for therapeutic strategy making, as patients with MCS generally show greater responses to some treatments (11).

In clinical practice, electroencephalography (EEG) recordings are often used as a tool to help clinicians with diagnoses and prognoses $(10,12)$. Analyses of resting-state EEG and event-related potential (ERP) are commonly employed (9). An ERP analysis objectively examines sensory and cognitive functions by averaging repeated stimulus-evoked EEG activity $(2,13)$. Several passive and active paradigms have been used in patients with DOC (13-17). However, the passive paradigms, such as mismatch negativity and somatosensory-evoked potentials, are overly dependent on the basic perceptional function and cortical sensors, which are commonly less preserved in DOC patients 
following severe brain injuries $(2,18)$. The active paradigms, such as motor imagery, require the active participation of patients. This poses several problems when working with DOC patients, such as their impaired cognitive function, fluctuations of arousal levels, fatigue, and subclinical seizure activity. EEG recordings taken in a resting state denote spontaneous neural activity, which is relevant to the fundamental brain state $(3,19)$. Therefore, appropriate features derived from resting-sate EEG may be helpful in monitoring the brain condition of DOC and contribute to decision-making related to these patients' care. In this paper, we present a review of studies on resting-state EEG in DOC and attempt to improve our knowledge of EEG features in the diagnosis, prognosis, and evaluation of brain interventions in cases of DOC.

\section{THE EEG ANALYSIS FOR DIAGNOSIS}

Since MCS patients are considered to benefit relatively easily from some specific treatments, compared to VS $(11,20)$, the ability to differentiate an MCS from a VS would offer great value in making decisions about treatment. In clinical practice, many standardized behavioral scales are used in the assessment of consciousness of brain-injured patients, such as the Glasgow Coma Scale (GCS) (21) and the Coma Recovery Scale-Revised (CRS-R) (22). Among them, the GCS is widely used in the early hours of a patient's admission, and the CRS-R is used throughout the recovery (1). However, a high ratio of misdiagnoses can be caused by clinicians' subjective judgments, motor function injuries, and patients' fluctuating levels of awareness $(10,16,23)$. Therefore, one of the primary applications of EEG studies in DOC patients is auxiliary diagnosis. Table 1 summarizes the studies we reviewed in this paper.

Spectrum powers have demonstrated the ability to discriminate between MCS and VS. VS patients have shown increased delta power but decreased alpha power, compared to those with MCS $(35,44)$. In comparison with healthy subjects, VS patients have shown higher delta and theta frequency powers, and both MCS and VS patients have shown decreased alpha power (39). Moreover, the ratios between higher frequencies (alpha + beta) and lower frequencies (delta + theta) have shown a positive correlation with patients' CRS-R scores $(24,39)$ and a correlation with regional glucose metabolism in MCS $(n=4)$ (24). Considering the spatial distribution, cortical EEG sources showed that the MCS and VS have significant variations of delta in the frontal region, theta in the frontal and parietal regions, alpha and beta in the central region, and gamma in the parietal region (43).

Spectral entropy analysis has found that the MCS has higher entropy value than the VS $(18,31)$, and the entropy values were correlated with CRS-R (31). The spectral entropy of the MCS changes over time, and periodicities closely resemble being awake in healthy subjects (44). Therefore, the spectral entropy value and its periodic characteristic have been suggested as potential indices for differentiating the MCS from VS. Some other spectrum-derived indices have been introduced in DOC research, such as BIS. BIS was demonstrated to discriminate between an unconscious state and a conscious one (with a value of 50) in one study (25). It could effectively distinguish the VS from the MCS (26).
Entropy theory has also been applied in the time domain of EEG. Approximate entropy (28-30), Lempel-Ziv complexity (30), permutation entropy (18), and Kolmogorov-Chaitin complexity (18) indices have been proposed to investigate the association of EEG complexity with the consciousness levels of DOC patients. Generally, the VS had lower EEG complexity than the MCS, and the control had the highest (30). Among the indices, Kolmogorov-Chaitin complexity and permutation entropy have been indicated as capable of discriminating the MCS from the VS $(18,45)$.

Functional connectivity is a crucial method for examining consciousness $(40,67)$. Among the connectivity methods, coherence was the earliest connectivity measurement used in DOC research (62). The results of one study showed that the frontal regions and their connections with the left temporal and parietooccipital areas could differentiate the MCS and severe neurocognitive disorders, and this difference was consistent with the results of a Granger causality (27). Similarly, a study of coherence performed by Leon-Carrion et al. showed significant differences in full bandwidth (delta, theta, alpha, and beta) in MCS patients with severe neurocognitive disorders (34). However, the coherence methodology has inherent defects that prevent it from being considered as an ideal method for describing global networks $(68,69)$. Lehembre et al. compared three connectivity methods (coherence, the imaginary part of coherence, and the phase lag index) and found that significantly lower connectivity of the VS than the MCS could be detected by the imaginary part of coherence and the phase lag index, but failed with coherence (35). Another study addressed 44 indices and proved that partial coherence, directed transfer function, and generalized partial directed coherence were methods with above-chance accuracy for the distinction of an MCS from a VS (with accuracy levels of $0.88,0.80$, and 0.78 , respectively) (40).

Furthermore, some other connectivity approaches have been employed, such as weighted symbolic mutual information (wSMI), cross-approximate entropy (32), debiased weighted phase lag index (dwPLI) (46), symbolic transfer entropy, and multivariate Granger causality (41). Among them, wSMI has demonstrated a dissociation with consciousness levels in DOC patients (36), and it was significantly lower in VS in theta and alpha bands (18). Similarly, connectivity and network parameters measured by dwPLI in delta and alpha bands also provided valuable approaches to discriminate different consciousness levels in DOC patients (46).

New approaches using non-strict resting-state EEG might provide new perspectives for finding physiological features that may contribute to diagnoses. Standard EEG patterns in DOC patients showed a difference between the MCS and VS in sleeping states (33). The occurrence of EEG patterns, including sleep spindles, slow wave activity, and the variability of brain rhythms (theta, alpha, and beta), were demonstrated to have significant correlations with the patients' behavioral diagnoses (37). Bonfiglio et al. proposed that the detection of blink-rated oscillations contributed to the differential diagnoses between the MCS and VS $(38,42)$. Blink-related delta oscillations linked with awareness of the surrounding environment, which was a criterion for assessing consciousness. The detection of blink-related activity differs 
TABLE 1 | Summary of studies using resting-state EEG for diagnosis, prognosis, and evaluation of intervention and basic researches.

\begin{tabular}{|c|c|c|c|c|c|}
\hline Objectives & Literatures & Methods & Subjects & Accuracy/sensitivity/specificity (\%) & Main results \\
\hline \multirow[t]{23}{*}{ Diagnosis } & Coleman et al. (24) & Spectrum power ratio & MCS 4, VS 6 & $-/-/-$ & VS showed significantly higher EEG power ratio than MCS \\
\hline & Schnakers et al. (25) & BIS & VS 32, Coma 11 & $-/ 75 / 75$ & BIS could differentiate unconscious from conscious \\
\hline & Schnakers et al. (26) & & $\begin{array}{l}\text { EMCS } 13 \text {, MCS } 30 \\
\text { VS } 13 \text {, Coma } 16\end{array}$ & $-/-/-$ & \\
\hline & Pollonini et al. (27) & Coherence, Granger causality & MCS 7, SND 9 & $100 /-/-$ & $\begin{array}{l}\text { Number of connections within and between brain regions } \\
\text { could differentiate MCS from SND }\end{array}$ \\
\hline & Sara and Pistoia (28) & ApEn & VS 10 , control 10 & $-/-/-$ & ApEn was lower in VS than in controls \\
\hline & Sarà et al. (29) & & VS 38 , control 40 & $-/ 100 / 97.5$ & \\
\hline & Wu et al. (30) & $\begin{array}{l}\text { Lempel-Ziv complexity, ApEn, cross- } \\
\text { approximate entropy }\end{array}$ & MCS 16, VS 21, control 30 & $-/-/-$ & $\begin{array}{l}\text { VS had lowest non-linear indices than MCS and control had } \\
\text { highest indices }\end{array}$ \\
\hline & Gosseries et al. (31) & State entropy, response entropy & MCS 26, VS 24, Coma 6 & $-/ 89 / 90$ & EEG entropy of MCS was higher than VS \\
\hline & Wu et al. (32) & Cross-approximate entropy & MCS 20, VS 30, control 30 & $-/-/-$ & $\begin{array}{l}\text { Interconnection of local and distant cortical networks in MCS } \\
\text { was superior to that of VS }\end{array}$ \\
\hline & Landsness et al. (33) & Slow wave activity & MCS 6, VS 5 & $-/-/-$ & $\begin{array}{l}\text { MCS showed an alternating sleep pattern; } \\
\text { VS preserved behavioral sleep but no sleep EEG patterns; }\end{array}$ \\
\hline & Leon-Carrion et al. (34) & Coherence, Granger causality & MCS 7, SND 9 & $-/-/-$ & $\begin{array}{l}\text { MCS showed frontal cortex disconnection from other cortical } \\
\text { regions }\end{array}$ \\
\hline & & & & & $\begin{array}{l}\text { Significant difference in full bandwidth coherence between } \\
\text { SND and MCS }\end{array}$ \\
\hline & Lehembre et al. (35) & $\begin{array}{l}\text { Spectrum power, coherence, imaginary } \\
\text { part of coherence, phase lag index }\end{array}$ & $\begin{array}{l}\text { MCS 18, VS 10, } \\
\text { Acute/subacute } 15\end{array}$ & $-/-/-$ & $\begin{array}{l}\text { VS showed increased delta, decreased alpha power, and } \\
\text { lower connectivity than MCS }\end{array}$ \\
\hline & King et al. (36) & wSMI & $\begin{array}{l}\text { MCS } 68 \text {, VS } 75 \text {, CS } 24 \\
\text { control } 14\end{array}$ & $-/-/-$ & WSMl increases as a function separate VS from MCS \\
\hline & Malinowska et al. (37) & $\begin{array}{l}\text { Matching pursuit decomposition, Slow } \\
\text { wave activity, K-complexes }\end{array}$ & LIS 1, MCS 20, VS 11 & $87 /-1-$ & Sleep EEG patterns correlated with patients' diagnosis \\
\hline & Bonfiglio et al. (38) & Blink-related delta oscillations & MCS 5, VS 4, control 12 & $-/-/-$ & Patients showed abnormal blink-related delta oscillations \\
\hline & Lechinger et al. (39) & Spectrum power & MCS 9, VS 8, control 14 & $-/-/-$ & $\begin{array}{l}\text { Ratios between frequencies (above } 8 \mathrm{~Hz} \text { ) and (below } 8 \mathrm{~Hz} \text { ) } \\
\text { correlated with CRS-R }\end{array}$ \\
\hline & Höller et al. (40) & A total of 44 indices & MCS 22, VS 27, control 23 & $\begin{array}{l}\text { Partial coherence: MCS vs. VS (88), control } \\
\text { vs. MCS (96), control vs. VS (98) }\end{array}$ & $\begin{array}{l}\text { Connectivity was crucial for determining the level of } \\
\text { consciousness }\end{array}$ \\
\hline & & & & $\begin{array}{l}\text { Transfer function: MCS vs. VS (80), control } \\
\text { vs. MCS (87), control vs. VS (84) }\end{array}$ & \\
\hline & & & & $\begin{array}{l}\text { Partial coherence: MCS vs. VS (78), control } \\
\text { vs. MCS (93), control vs. VS (96) }\end{array}$ & \\
\hline & Sitt et al. (18) & $\begin{array}{l}\text { Spectrum power, spectral entropy, } \\
\text { Kolmogorov-Chaitin complexity, phase } \\
\text { locking index, wSMI, permutation entropy }\end{array}$ & $\begin{array}{l}\text { MCS } 68, \text { VS } 75, \text { CS } 24 \\
\text { control } 14\end{array}$ & $\begin{array}{l}\text { Best cross-validated single measure: MCS vs } \\
\text { Whole set of measures: MCS vs. VS (AUC = } \\
\text { The most discriminative measure was wSMI, }\end{array}$ & $\begin{array}{l}\text { VS. VS }(A \cup C=71 \pm 4) \\
=78 \pm 4) \\
\text { I, which separated VS from MCS }\end{array}$ \\
\hline & Marinazzo et al. (41) & $\begin{array}{l}\text { Multivariate Granger causality, transfer } \\
\text { entropy }\end{array}$ & $\begin{array}{l}\text { MCS 10, EMCS 5, VS11, } \\
\text { control } 10\end{array}$ & $-/-/-$ & $\begin{array}{l}\text { In VS, the central, temporal, and occipital electrodes showed } \\
\text { asymmetry between incoming and outgoing information }\end{array}$ \\
\hline & Bonfiglio et al. (42) & $\begin{array}{l}\text { Blink-related synchronization/ } \\
\text { desynchronization }\end{array}$ & MCS 4, VS 5, control 12 & $-/-/-$ & $\begin{array}{l}\text { Blink-related synchronization/desynchronization could } \\
\text { differentiate MCS from VS }\end{array}$ \\
\hline
\end{tabular}


Prognosis Babiloni et al. (47) Wu et al. (30)

Fingelkurts et al. (48) EEG oscillatory microstates Sarà et al. (29) ApEn

Cologan et al. (49) Sleep spindles

Arnaldi et al. (50) Sleep patterns

Schorr et al. (51) Spectrum power, coherence

Wislowska et al. (52) Spectral power, sleep patterns, permutation entropy

Chennu et al. (46) dwPLI, brain network

Treatment evaluation

\section{Methods}

Naro et al. (43)

Piarulli et al. (44)

Thul et al. (45) Spectrum power, LORETA Spectrum power, spectral entropy

Permutation entropy, symbolic transfer entropy

\section{Subjects}

Accuracy/sensitivity/specificity (\%)

MCS 7, VS 6, control 10

MCS 6, VS 6

MCS 7, VS 8, control 24

8, control 24

Chennu et al. (46) dwPLI, brain network VS (Max AUC = 0.71) (Max AUC = 0.74)
Spectrum power, coherence, zolpidem

Manganotti et al. (54) Spectrum power, $20 \mathrm{~Hz}$ rTMS MCS 3, VS 3

Carboncini et al. (55) Spectrum power, phase synchronization, MCS 1 midazolam

Cavinato et al. (56) Coherence, simple sensory stimuli MCS 11, VS 15

\begin{tabular}{lll}
\hline Pisani et al. (57) & Slow wave activity, 5 Hz rTMS & MCS 4, VS 6 \\
\hline Naro et al. (58) & Spectrum power, coherence, tACS & MCS 12, VS 14, control 15
\end{tabular}

frontoparietal networks in all the control, partial MCS, and some VS

Naro et al. (59) Spectrum power, coherence, otDCS

MCS 10, VS 10, control 10 Fronto-parietal networks modulation, theta and gamma power modulation, and coherence increase were paralleled by a transient CRS-R improvement, only in MCS individuals

Naro et al. (60) Lagged-phase synchronization, network MCS 9, VS 11, control 10 Two VS patients showed a residual rTMS-induced modulation of the functional correlations between the

parameters, rTMS default mode network and the external awareness networks, as observed in MCS

Delta band connectivity and network had a clear relationship with outcomes

rTMS over M1 induced long-lasting behavioral and neurophysiological modifications in one MCS patient

Change in the power spectrum was observed after midazolam

Midazolam induced significant connectivity changes

Increase in short-range parietal and long-range fronto-parietal coherences in gamma frequencies was seen in the controls and MCS

VS showed no modifications in EEG patterns after stimulation

Following the real rTMS, a preserved sleep-wake cycle, a standard temporal progression of sleep stages appeared in all MCS but none of VS
Alpha was the most significant LORETA data correlating with consciousness level

MCS showed higher theta and alpha, lower delta, higher spectral entropy, and higher time variability than VS

Permutation entropy: Control vs. MCS (Max AUC $=0.74)$, control vs. VS (Max AUC $=0.91$ ), MCS vs. VS

政, VS vs. MCS: Alpha participation coefficient (AUC

(AU.82), alpha modular span $(\mathrm{AUC}=0.78)$

MCS - vs. MCS+: delta power averaged over all channels $(A \cup C=0.79)$

$\begin{array}{ll}\text { VS 50, control } 30 & \text { Power of alpha source predicted the follow-up recovery } \\ \text { MCS 16, VS 21, control } 30 & \text { Non-linear indices of patients who recovered increased than those in non-recovery }\end{array}$

MCS 11, VS $14 \quad$ Diversity and variability of EEG for non-survivors were significantly lower than for survivors

Patients with lowest ApEn either died or remained in VS, patients with highest ApEn became MCS or partial or full recovery

MCS 6, VS $20 \quad$ Sleep patterns were valuable predictors of a positive clinical outcome in sub-acute patients

MCS 15, VS 58, control 24 Short- and long-range coherence had a diagnostic value in the prognosis of recovery from VS

Day-night changes in EEG power spectra and signal complexity were revealed in MCS, but not VS Sleep patterns were linearly related to outcome 


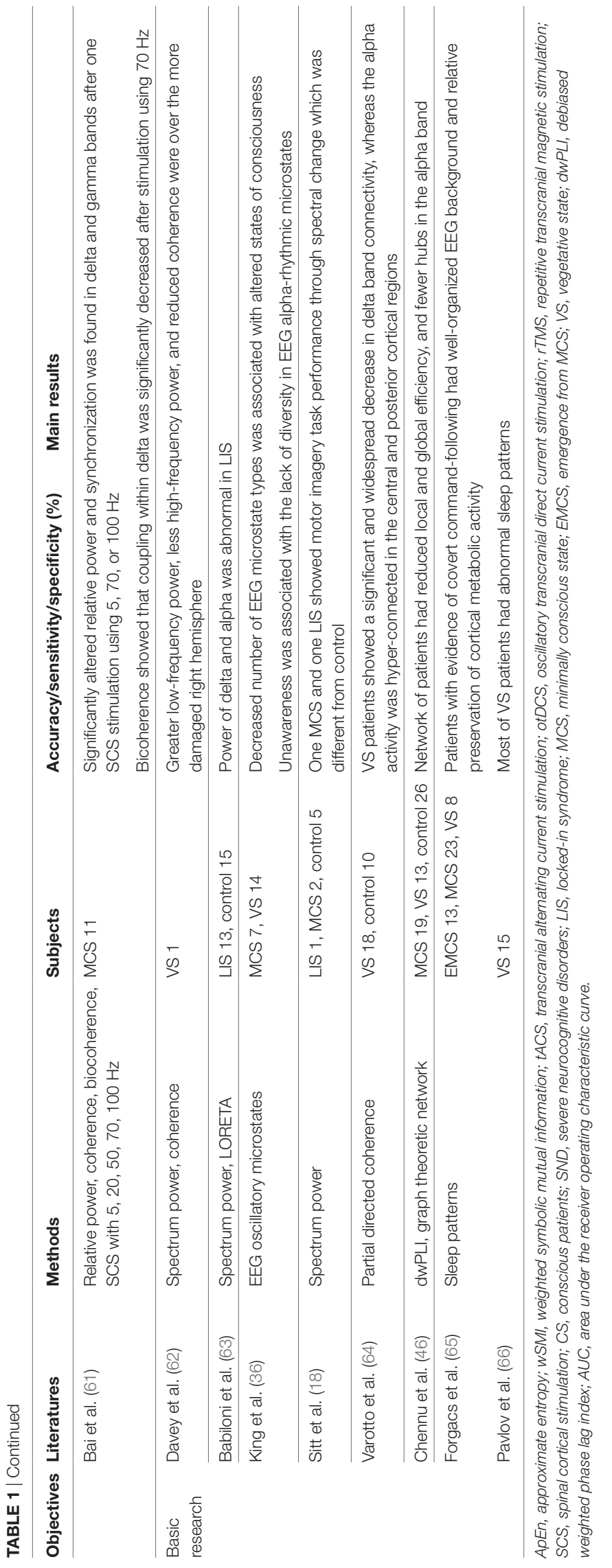

from the classical resting-state measurement. However, although it included an event input, the resting-state blinking used in the studies was also a type of spontaneous activity which differed from external stimulus used in ERP.

\section{THE EEG ANALYSIS FOR PROGNOSIS}

The prognosis for survival and recovery of DOC is still difficult under present clinical conditions (70,71). Generally, the outcomes at 3 and 6 months following the first assessment were selected to observe the predictive performance of the measures. After 3 months of observation, the spectrum power of EEG recordings showed potentially positive performance in predicting the outcomes of a persistent VS (a patient stays in a VS over 1 month after brain injury) (47). Measured by the level of the cognitive functioning scale (LCF), 12 of 50 patients recovered (from LCF III to LCF V-VIII). All the patients stayed in a chronic DOC state at the first evaluation. Compared to healthy subjects, the power of alpha in the occipital region showed progressive decay from healthy subjects to recovered patients and then to non-recovered patients. Therefore, the alpha oscillation was implied as a predictor of the possibility of consciousness recovery (47).

Studies using 6 months of observation have shown an association of non-linear analysis indices with the follow-up recovery. Lempel-Ziv complexity, ApEn, and cross-approximate entropy have been suggested as being capable of predicting outcomes of DOC patients (10 of 37 recovered, with Glasgow Outcome Scale scores decreasing to 3 ). The first evaluation was conducted on patients who stayed in a chronic DOC state after the onset of brain injury for less than 6 months; the patients with increasing indices under painful stimuli had a higher probability of recovery (30). Coincidentally, another study also found that the highest ApEn might correspond to partial or full consciousness improvement at 6 months after the first assessment (29). The prognostic value of resting-state EEG in predicting survival or non-survival 6 months after brain injury was also proven by EEG oscillatory microstate analyses (48). The first EEG recording of the patients was obtained between 14 days and 3 months after acute brain events. The diversity and variability of EEG oscillations and the probability of the appearance of delta, theta (slow and fast), and alpha oscillations were shown to be potential prognostic features in predicting the outcomes of DOC at the group level. In a recent study, 39 of 61 patients had positive outcomes (assessed by Glasgow Outcome Scale-Extended) at 1 year following the first assessment (46). EEG analysis of the patients found that the connectivity and brain network parameters in delta band had a clear relationship with their outcomes. Meanwhile, EEG sleep patterns were also demonstrated valuable predictors of patients' clinical outcomes $(49,50,52)$. Especially, the density of sleep spindles provided significantly predictive and valuable information about the clinical outcomes of DOC patients.

\section{THE EEG ANALYSIS FOR THE EVALUATION OF BRAIN INTERVENTION}

Due to a variety of etiological, brain injury, and cortical conditions, DOC patients have shown various responses to treatment 


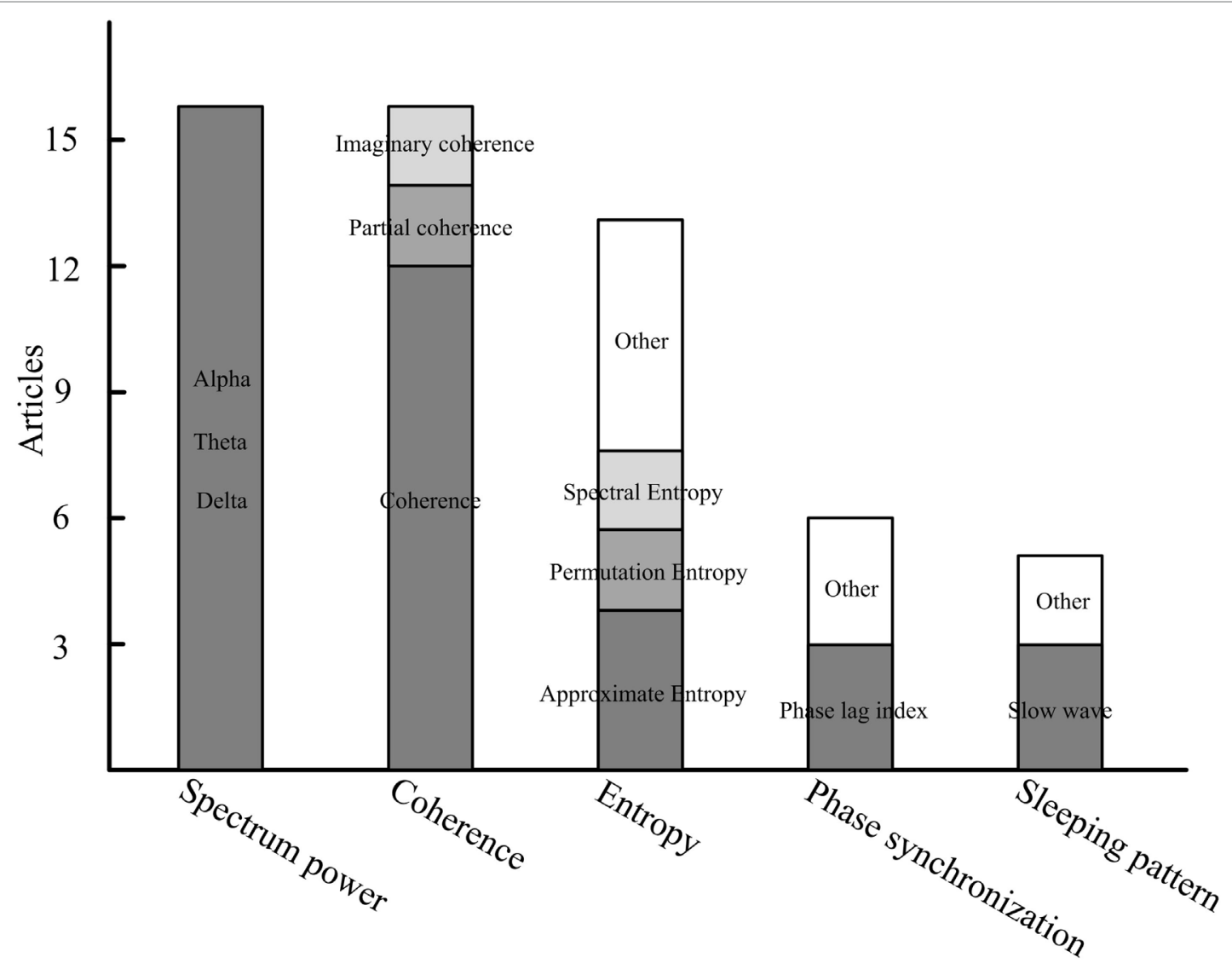

FIGURE 1 | The primary features in resting-state electroencephalography studies of disorders of consciousness.

therapies $(20,72)$. A precise evaluation of the cerebral responses in the treatment would be helpful for understanding the mechanism of the intervention and facilitate the creation of individual therapeutic strategies. In practice, behavioral changes induced by treatment might be long-lasting accumulated effects that could not be observed immediately. Recently, indices based on EEG analyses were applied to monitor the instantaneous cerebral responses in pharmacological and non-pharmacological brain interventions (16).

Spectrum power, connectivity of coherence, and phase synchronization have been used to assess the cerebral changes of patients in pharmacological treatment $(53,55)$. For MCS patients who respond to midazolam, spectrum power changes and connectivity changes were found after taking the medication (55). While under zolpidem treatment, all patients showed a distinct low-frequency oscillatory peak at approximately 6-10 $\mathrm{Hz}$ over the fronto-central regions (53). Resting-state EEG in nonpharmacological interventions have been investigated in DOC treatment, such as spinal cord stimulation (61), repetitive transcranial magnetic stimulation (rTMS) $(54,57,60)$, sensory stimuli (56), transcranial alternating current stimulation (58), and oscillatory transcranial direct current stimulation (59). The fronto-parietal networks of the MCS in the theta and gamma bands have been demonstrated as being responsive to transcranial current stimulation, with little reactivity found in the VS $(58,59)$. This modulation of a consciousness-related network may suggest more benefits to the MCS than the VS from transcranial current stimulation, and the differential cortical responses between the MCS and VS might provide a stimulus-response approach for diagnoses. Similarly, the different EEG responses between the MCS and VS have also been demonstrated in rTMS, proven by spectrum power (54), complex network parameters (60), and slow wave activity in sleeping (57). In addition, we have attempted to use resting-state EEG as an assistive method for parameter selection in spinal cord stimulations of patients with DOC (61).

\section{SUMMARY AND CONCLUSION}

The characteristics that have been applied in DOC-related studies could be generally classified into five categories: the spectrum, entropy, connectivity, the network, and the sleeping pattern. We summarize the primary features that are frequently used in DOC studies (Figure 1). We found that spectrum power, coherence, and entropy were the most frequently used features in differentiating consciousness levels, predicting follow-up outcome or measuring patients' cortical response to brain intervention. Comparisons of various methods with multiple indices were performed in two studies $(18,40)$. Indices derived from spectrum, non-linear 
analysis, information theory, and functional connectivity were investigated. A discrimination performance of the measures supports power spectrum and functional connectivity as having the best performance in separating the VS from the MCS and healthy subjects (18). In addition, permutation entropy in the theta frequency also has relatively higher classification accuracy in distinguishing the MCS and VS.

Spectral power measures the strength of neuronal oscillations, which depend on the spontaneously activity of underlying oscillators (neurons) (73). Spectral power at some specific frequency can reveal relationships between the activity of groups of neurons and consciousness levels $(24,39)$. Reviewing the studies, increases of low power (delta and theta), and decreases of high power (alpha) were common spectrum characteristics of patients with DOC. In comparing the MCS and VS, the latter has increased delta and decreased alpha power than the former. Therefore, a power ratio index may be first considered to help us qualitatively assess the consciousness state of patients. In predicting the follow-up outcomes, alpha power should always receive attention. In addition, theta and alpha bands are also critical frequency bands in assessing cortical responses to brain interventions.

Since the neuronal oscillations and synchronization are two essential features of the conscious brain (74), synchronization should be a critical feature in understanding the consciousness of patients with DOC. Synchronization analysis could reveal direct structural connections or indirect information flows, and it could concurrently provide temporal causality and spatial links $(2,75)$. Non-directed (coherence, the phase locking index, partial directed coherence, the imaginary part of coherence, the dwPLI, cross-approximate entropy, and wSMI) and directed (transfer entropy, symbolic transfer entropy, mutual information, and Granger causality) connectivity measurements were used to reveal the "disconnection" characteristics of patients with DOC $(32,64)$. Among the measurements, coherence is the most commonly used method. In addition, disconnection between the frontal and other regions, especially the fronto-parietal, was shown to be a

\section{REFERENCES}

1. Gosseries O, Vanhaudenhuyse A, Bruno M-A, Demertzi A, Schnakers C, Boly MM, et al. Disorders of consciousness: coma, vegetative and minimally conscious states. In: Cvetkovic D., Cosic I., editors. States of Consciousness. Berlin: Springer (2011). p. 29-55

2. Gosseries O, Di H, Laureys S, Boly M. Measuring consciousness in severely damaged brains. Annu Rev Neurosci (2014) 37:457-78. doi:10.1146/annurevneuro-062012-170339

3. Giacino JT, Fins JJ, Laureys S, Schiff ND. Disorders of consciousness after acquired brain injury: the state of the science. Nat Rev Neurol (2014) 10(2): 99-114. doi:10.1038/nrneurol.2013.279

4. Jennett B, Plum F. Persistent vegetative state after brain damage. A syndrome in search of a name. Lancet (1972) 1(7753):734-7. doi:10.1016/ S0140-6736(72)90242-5

5. Laureys S, Celesia GG, Cohadon F, Lavrijsen J, Leon-Carrion J, Sannita WG, et al. Unresponsive wakefulness syndrome: a new name for the vegetative state or apallic syndrome. BMC Med (2010) 8:68. doi:10.1186/1741-7015-8-68

6. Laureys S, Owen AM, Schiff ND. Brain function in coma, vegetative state, and related disorders. Lancet Neurol (2004) 3(9):537-46. doi:10.1016/S14744422(04)00852-X significant biomarker, whether assessing the consciousness level or evaluating the brain response to intervention. However, when taking the synchronization feature into actual clinical operation, the reference location, artifact robustness, volume conduction, interesting regions, and cautious physiological explanations should be taken into account.

Similar to EEG complexity in a sleep or anesthesia state (76-78), the complexity measures in DOC were based on the hypothesis that neural activities would be suppressed in a brain of a low consciousness level, and thus fewer components would be included in the EEG signals. EEG complexity, whether measured in the time domain (such as approximate entropy, Lempel-Ziv complexity, Kolmogorov-Chaitin complexity, and permutation entropy) or the frequency domain (BIS and spectral entropy), provided relatively effective and readily comprehensible indices (range of $0-1$ or $0-100$, with higher value corresponding to higher consciousness level) to describe brain electrical activities under different consciousness states. Therefore, complexity characteristics may have potential value in quantitatively describing the consciousness level of patients with DOC and finally are implanted into monitors for daily caring.

\section{AUTHOR CONTRIBUTIONS}

YB and XX reviewed the articles and written the manuscript. XL guided the whole work.

\section{FUNDING}

This research was supported by the National Natural Science Foundation of China (No. 61273063, No. 81230023) and the Beijing Municipal Science and Technology Commission (No. Z141107002514111), and the commercialization of research findings was supported by the Beijing Municipal Commission of Education.

7. Laureys S, Boly M. The changing spectrum of coma. Nat Clin Pract Neurol (2008) 4(10):544-6. doi:10.1038/ncpneuro0887

8. Giacino JT, Ashwal S, Childs N, Cranford R, Jennett B, Katz DI, et al. The minimally conscious state: definition and diagnostic criteria. Neurology (2002) 58(3):349-53. doi:10.1212/WNL.58.3.349

9. Cruse D, Monti MM, Owen AM. Neuroimaging in disorders of consciousness: contributions to diagnosis and prognosis. Future Neurol (2011) 6(2):291-9. doi:10.2217/fnl.10.87

10. Gantner IS, Bodart O, Laureys S, Demertzi A. Our rapidly changing understanding of acute and chronic disorders of consciousness: challenges for neurologists. Future Neurol (2013) 8(1):43-54. doi:10.2217/fnl.12.77

11. Thibaut A, Bruno MA, Ledoux D, Demertzi A, Laureys S. tDCS in patients with disorders of consciousness: sham-controlled randomized double-blind study. Neurology (2014) 82(13):1112-8. doi:10.1212/WNL. 0000000000000260

12. Fernandez-Espejo D, Owen AM. Detecting awareness after severe brain injury. Nat Rev Neurosci (2013) 14(11):801-9. doi:10.1038/nrn3608

13. Cruse D, Chennu S, Chatelle C, Bekinschtein TA, Fernandez-Espejo D, Pickard JD, et al. Bedside detection of awareness in the vegetative state: a cohort study. Lancet (2011) 378(9809):2088-94. doi:10.1016/S0140-6736(11)61224-5

14. Cruse D, Chennu S, Fernandez-Espejo D, Payne WL, Young GB, Owen AM. Detecting awareness in the vegetative state: electroencephalographic evidence 
for attempted movements to command. PLoS One (2012) 7(11):e49933. doi:10.1371/journal.pone.0049933

15. Cruse D, Beukema S, Chennu S, Malins JG, Owen AM, McRae K. The reliability of the N400 in single subjects: implications for patients with disorders of consciousness. Neuroimage Clin (2014) 4:788-99. doi:10.1016/j.nicl.2014.05.001

16. Gosseries O, Pistoia F, Charland-Verville V, Carolei A, Sacco S, Laureys S. The role of neuroimaging techniques in establishing diagnosis, prognosis and therapy in disorders of consciousness. Open Neuroimag J (2016) 10:52-68. doi:10.2174/1874440001610010052

17. Monti MM, Sannita WG. Brain Function and Responsiveness in Disorders of Consciousness. Switzerland: Springer International Publishing (2016).

18. Sitt JD, King JR, El Karoui I, Rohaut B, Faugeras F, Gramfort A, et al. Large scale screening of neural signatures of consciousness in patients in a vegetative or minimally conscious state. Brain (2014) 137(Pt 8):2258-70. doi:10.1093/ brain/awu141

19. Stam C, Montez T, Jones B, Rombouts S, Van Der Made Y, Pijnenburg Y, et al. Disturbed fluctuations of resting state EEG synchronization in Alzheimer's disease. Neurophysiol Clin (2005) 116(3):708-15. doi:10.1016/j. clinph.2004.09.022

20. Guerra A, Costantini EM, Maatta S, Ponzo D, Ferreri F. Disorders of consciousness and electrophysiological treatment strategies: a review of the literature and new perspectives. Curr Pharm Des (2014) 20(26):4248-67. doi: $10.2174 / 13816128113196660648$

21. Teasdale G, Jennett B. Assessment of coma and impaired consciousness. A practical scale. Lancet (1974) 2(7872):81-4. doi:10.1016/S01406736(74)91639-0

22. Giacino JT, Kalmar K, Whyte J. The JFK Coma Recovery Scale-revised: measurement characteristics and diagnostic utility. Arch Phys Med Rehabil (2004) 85(12):2020-9. doi:10.1016/j.apmr.2004.02.033

23. Schnakers C, Vanhaudenhuyse A, Giacino J, Ventura M, Boly M, Majerus S, et al. Diagnostic accuracy of the vegetative and minimally conscious state: clinical consensus versus standardized neurobehavioral assessment. BMC Neurol (2009) 9:35. doi:10.1186/1471-2377-9-35

24. Coleman MR, Menon DK, Fryer TD, Pickard JD. Neurometabolic coupling in the vegetative and minimally conscious states: preliminary findings. J Neurol Neurosurg Psychiatry (2005) 76(3):432-4. doi:10.1136/jnnp.2004.045930

25. Schnakers C, Majerus S, Laureys S. Bispectral analysis of electroencephalogram signals during recovery from coma: preliminary findings. Neuropsychol Rehabil (2005) 15(3-4):381-8. doi:10.1080/09602010443000524

26. Schnakers C, Ledoux D, Majerus S, Damas P, Damas F, Lambermont B, et al. Diagnostic and prognostic use of bispectral index in coma, vegetative state and related disorders. Brain Inj (2008) 22(12):926-31. doi:10.1080/ 02699050802530565

27. Pollonini L, Pophale S, Situ N, Wu MH, Frye RE, Leon-Carrion J, et al. Information communication networks in severe traumatic brain injury. Brain Topogr (2010) 23(2):221-6. doi:10.1007/s10548-010-0139-9

28. Sara M, Pistoia F. Complexity loss in physiological time series of patients in a vegetative state. Nonlinear Dynamics Psychol Life Sci (2010) 14(1):1-13.

29. Sarà M, Pistoia F, Pasqualetti P, Sebastiano F, Onorati P, Rossini PM. Functional isolation within the cerebral cortex in the vegetative state: a nonlinear method to predict clinical outcomes. Neurorehabil Neural Repair (2011) 25(1):35-42. doi:10.1177/1545968310378508

30. Wu DY, Cai G, Yuan Y, Liu L, Li GQ, Song WQ, et al. Application of nonlinear dynamics analysis in assessing unconsciousness: a preliminary study. Clin Neurophysiol (2011) 122(3):490-8. doi:10.1016/j.clinph.2010.05.036

31. Gosseries O, Schnakers C, Ledoux D, Vanhaudenhuyse A, Bruno MA, Demertzi A, et al. Automated EEG entropy measurements in coma, vegetative state/unresponsive wakefulness syndrome and minimally conscious state. Funct Neurol (2011) 26(1):25-30.

32. Wu DY, Cai G, Zorowitz RD, Yuan Y, Wang J, Song WQ. Measuring interconnection of the residual cortical functional islands in persistent vegetative state and minimal conscious state with EEG nonlinear analysis. Clin Neurophysiol (2011) 122(10):1956-66. doi:10.1016/j.clinph.2011.03.018

33. Landsness E, Bruno MA, Noirhomme Q, Riedner B, Gosseries O, Schnakers C, et al. Electrophysiological correlates of behavioural changes in vigilance in vegetative state and minimally conscious state. Brain (2011) 134(Pt 8): 2222-32. doi:10.1093/brain/awr152

34. Leon-Carrion J, Leon-Dominguez U, Pollonini L, Wu M-H, Frye RE, Dominguez-Morales MR, et al. Synchronization between the anterior and posterior cortex determines consciousness level in patients with traumatic brain injury (TBI). Brain Res (2012) 1476:22-30. doi:10.1016/j. brainres.2012.03.055

35. Lehembre R, Marie-Aurelie B, Vanhaudenhuyse A, Chatelle C, Cologan V, Leclercq Y, et al. Resting-state EEG study of comatose patients: a connectivity and frequency analysis to find differences between vegetative and minimally conscious states. Funct Neurol (2012) 27(1):41-7.

36. King JR, Sitt JD, Faugeras F, Rohaut B, El Karoui I, Cohen L, et al. Information sharing in the brain indexes consciousness in noncommunicative patients. Curr Biol (2013) 23(19):1914-9. doi:10.1016/j.cub.2013.07.075

37. Malinowska U, Chatelle C, Bruno M-A, Noirhomme Q, Laureys S, Durka PJ. Electroencephalographic profiles for differentiation of disorders of consciousness. Biomed Eng Online (2013) 12(1):109. doi:10.1186/1475-925X-12-109

38. Bonfiglio L, Olcese U, Rossi B, Frisoli A, Arrighi P, Greco G, et al. Cortical source of blink-related delta oscillations and their correlation with levels of consciousness. Hum Brain Mapp (2013) 34(9):2178-89. doi:10.1002/hbm. 22056

39. Lechinger J, Bothe K, Pichler G, Michitsch G, Donis J, Klimesch W, et al. CRS-R score in disorders of consciousness is strongly related to spectral EEG at rest. J Neurol (2013) 260(9):2348-56. doi:10.1007/s00415-013-6982-3

40. Höller Y, Thomschewski A, Bergmann J, Kronbichler M, Crone JS, Schmid EV, et al. Connectivity biomarkers can differentiate patients with different levels of consciousness. Clin Neurophysiol (2014) 125(8):1545-55. doi:10.1016/j. clinph.2013.12.095

41. Marinazzo D, Gosseries O, Boly M, Ledoux D, Rosanova M, Massimini M, et al. Directed information transfer in scalp electroencephalographic recordings: insights on disorders of consciousness. Clin EEG Neurosci (2014) 45(1):33-9. doi:10.1177/1550059413510703

42. Bonfiglio L, Piarulli A, Olcese U, Andre P, Arrighi P, Frisoli A, et al. Spectral parameters modulation and source localization of blink-related alpha and low-beta oscillations differentiate minimally conscious state from vegetative state/unresponsive wakefulness syndrome. PLoS One (2014) 9(3):e93252. doi:10.1371/journal.pone.0093252

43. Naro A, Bramanti P, Leo A, Cacciola A, Bramanti A, Manuli A, et al. Towards a method to differentiate chronic disorder of consciousness patients' awareness: the low-resolution brain electromagnetic tomography analysis. J Neurol Sci (2016) 368:178-83. doi:10.1016/j.jns.2016.07.016

44. Piarulli A, Bergamasco M, Thibaut A, Cologan V, Gosseries O, Laureys S. EEG ultradian rhythmicity differences in disorders of consciousness during wakefulness. J Neurol (2016) 263(9):1746-60. doi:10.1007/s00415-016-8196-y

45. Thul A, Lechinger J, Donis J, Michitsch G, Pichler G, Kochs EF, et al. EEG entropy measures indicate decrease of cortical information processing in disorders of consciousness. Neurophysiol Clin (2016) 127(2):1419-27. doi:10.1016/j.clinph.2015.07.039

46. Chennu S, Annen J, Wannez S, Thibaut A, Chatelle C, Cassol H, et al. Brain networks predict metabolism, diagnosis and prognosis at the bedside in disorders of consciousness. Brain (2017) 140(8):2120-32. doi:10.1093/brain/ awx 163

47. Babiloni C, Sara M, Vecchio F, Pistoia F, Sebastiano F, Onorati P, et al. Cortical sources of resting-state alpha rhythms are abnormal in persistent vegetative state patients. Clin Neurophysiol (2009) 120(4):719-29. doi:10.1016/j.clinph. 2009.02.157

48. Fingelkurts AA, Fingelkurts AA, Bagnato S, Boccagni C, Galardi G. Life or death: prognostic value of a resting EEG with regards to survival in patients in vegetative and minimally conscious states. PLoS One (2011) 6(10):e25967. doi:10.1371/journal.pone.0025967

49. Cologan V, Drouot X, Parapatics S, Delorme A, Gruber G, Moonen G, et al. Sleep in the unresponsive wakefulness syndrome and minimally conscious state. J Neurotrauma (2013) 30(5):339-46. doi:10.1089/neu.2012.2654

50. Arnaldi D, Terzaghi M, Cremascoli R, De Carli F, Maggioni G, Pistarini C, et al. The prognostic value of sleep patterns in disorders of consciousness in the sub-acute phase. Clin Neurophysiol (2016) 127(2):1445-51. doi:10.1016/ j.clinph.2015.10.042

51. Schorr B, Schlee W, Arndt M, Bender A. Coherence in resting-state EEG as a predictor for the recovery from unresponsive wakefulness syndrome. J Neurol (2016) 263(5):937-53. doi:10.1007/s00415-016-8084-5

52. Wislowska M, Giudice RD, Lechinger J, Wielek T, Heib DP, Pitiot A, et al. Night and day variations of sleep in patients with disorders of consciousness. Sci Rep (2017) 7(1):266. doi:10.1038/s41598-017-00323-4 
53. Williams ST, Conte MM, Goldfine AM, Noirhomme Q, Gosseries O, Thonnard $\mathrm{M}$, et al. Common resting brain dynamics indicate a possible mechanism underlying zolpidem response in severe brain injury. Elife (2013) 2:e01157. doi:10.7554/eLife.01157

54. Manganotti P, Formaggio E, Storti SF, Fiaschi A, Battistin L, Tonin P, et al. Effect of high-frequency repetitive transcranial magnetic stimulation on brain excitability in severely brain-injured patients in minimally conscious or vegetative state. Brain Stimul (2013) 6(6):913-21. doi:10.1016/j. brs.2013.06.006

55. Carboncini MC, Piarulli A, Virgillito A, Arrighi P, Andre P, Tomaiuolo F, et al. A case of post-traumatic minimally conscious state reversed by midazolam: clinical aspects and neurophysiological correlates. Restor Neurol Neurosci (2014) 32(6):767-87. doi:10.3233/RNN-140426

56. Cavinato M, Genna C, Manganotti P, Formaggio E, Storti SF, Campostrini S, et al. Coherence and consciousness: study of fronto-parietal gamma synchrony in patients with disorders of consciousness. Brain Topogr (2015) 28(4):570-9. doi:10.1007/s10548-014-0383-5

57. Pisani LR, Naro A, Leo A, Arico I, Pisani F, Silvestri R, et al. Repetitive transcranial magnetic stimulation induced slow wave activity modification: a possible role in disorder of consciousness differential diagnosis? Conscious Cogn (2015) 38:1-8. doi:10.1016/j.concog.2015.09.012

58. Naro A, Bramanti P, Leo A, Russo M, Calabrò RS. Transcranial alternating current stimulation in patients with chronic disorder of consciousness: a possible way to cut the diagnostic Gordian knot? Brain Topogr (2016) 29(4):623-44. doi:10.1007/s10548-016-0489-Z

59. Naro A, Russo M, Leo A, Cannavò A, Manuli A, Bramanti A, et al. Cortical connectivity modulation induced by cerebellar oscillatory transcranial direct current stimulation in patients with chronic disorders of consciousness: a marker of covert cognition? Neurophysiol Clin (2016) 127(3):1845-54. doi:10.1016/j.clinph.2015.12.010

60. Naro A, Leo A, Manuli A, Cannavò A, Bramanti A, Bramanti P, et al. How far can we go in chronic disorders of consciousness differential diagnosis? The use of neuromodulation in detecting internal and external awareness. Neuroscience (2017) 349:165-73. doi:10.1016/j.neuroscience.2017.02.053

61. Bai Y, Xia X, Li X, Wang Y, Yang Y, Liu Y, et al. Spinal cord stimulation modulates frontal delta and gamma in patients of minimally consciousness state. Neuroscience (2017) 346:247-54. doi:10.1016/j.neuroscience. 2017.01.036

62. Davey MP, Victor JD, Schiff ND. Power spectra and coherence in the EEG of a vegetative patient with severe asymmetric brain damage. Clin Neurophysiol (2000) 111(11):1949-54. doi:10.1016/S1388-2457(00)00435-1

63. Babiloni C, Pistoia F, Sara M, Vecchio F, Buffo P, Conson M, et al. Resting state eyes-closed cortical rhythms in patients with locked-in-syndrome: an EEG study. Clin Neurophysiol (2010) 121(11):1816-24. doi:10.1016/j. clinph.2010.04.027

64. Varotto G, Fazio P, Rossi Sebastiano D, Duran D, D’Incerti L, Parati E, et al. Altered resting state effective connectivity in long-standing vegetative state patients: an EEG study. Clin Neurophysiol (2014) 125(1):63-8. doi:10.1016/j. clinph.2013.06.016

65. Forgacs PB, Conte MM, Fridman EA, Voss HU, Victor JD, Schiff ND. Preservation of electroencephalographic organization in patients with impaired consciousness and imaging-based evidence of command-following. Ann Neurol (2014) 76(6):869-79. doi:10.1002/ana.24283
66. Pavlov YG, Gais S, Muller F, Schonauer M, Schapers B, Born J, et al. Night sleep in patients with vegetative state. J Sleep Res (2017) 7:266. doi:10.1111/ jsr. 12524

67. Sanders RD, Tononi G, Laureys S, Sleigh JW. Unresponsiveness $\neq$ unconsciousness. J Am Soc Anesthesiol (2012) 116(4):946-59. doi:10.1097/ALN. 0b013e318249d0a7

68. Fein G, Raz J, Brown FF, Merrin EL. Common reference coherence data are confounded by power and phase effects. Electroencephalogr Clin Neurophysiol (1988) 69(6):581-4. doi:10.1016/0013-4694(88)90171-X

69. Srinivasan R, Nunez PL, Silberstein RB. Spatial filtering and neocortical dynamics: estimates of EEG coherence. IEEE Trans Biomed Eng (1998) 45(7):814-26. doi:10.1109/10.686789

70. Giacino JT, Kalmar K. Diagnostic and prognostic guidelines for the vegwive and minimally conscious states. Neuropsychol Rehabil (2005) 15(3-4):166-74. doi:10.1080/09602010443000498

71. Bagnato S, Boccagni C, Prestandrea C, Sant'Angelo A, Castiglione A, Galardi G. Prognostic value of standard EEG in traumatic and non-traumatic disorders of consciousness following coma. Neurophysiol Clin (2010) 121(3):274-80. doi:10.1016/j.clinph.2009.11.008

72. Georgiopoulos M, Katsakiori P, Kefalopoulou Z, Ellul J, Chroni E, Constantoyannis C. Vegetative state and minimally conscious state: a review of the therapeutic interventions. Stereotact Funct Neurosurg (2010) 88(4): 199-207. doi:10.1159/000314354

73. Buzsaki G, Draguhn A. Neuronal oscillations in cortical networks. Science (2004) 304(5679):1926-9. doi:10.1126/science.1099745

74. Ward LM. Synchronous neural oscillations and cognitive processes. Trends Cogn Sci (2003) 7(12):553-9. doi:10.1016/j.tics.2003.10.012

75. Boly M, Massimini M, Garrido MI, Gosseries O, Noirhomme Q, Laureys S, et al. Brain connectivity in disorders of consciousness. Brain Connect (2012) 2(1):1-10. doi:10.1089/brain.2011.0049

76. Acharya R, Faust O, Kannathal N, Chua T, Laxminarayan S. Non-linear analysis of EEG signals at various sleep stages. Comput Methods Programs Biomed (2005) 80(1):37-45. doi:10.1016/j.cmpb.2005.06.011

77. Li X, Cui S, Voss LJ. Using permutation entropy to measure the electroencephalographic effects of sevoflurane. Anesthesiology (2008) 109(3):448-56. doi:10.1097/ALN.0b013e318182a91b

78. Li D, Li X, Liang Z, Voss LJ, Sleigh JW. Multiscale permutation entropy analysis of EEG recordings during sevoflurane anesthesia. J Neural Eng (2010) 7(4):046010. doi:10.1088/1741-2560/7/4/046010

Conflict of Interest Statement: The authors declare that the research was conducted in the absence of any commercial or financial relationships that could be construed as a potential conflict of interest.

The reviewer, QN, and handling editor declared their shared affiliation.

Copyright (C) 2017 Bai, Xia and Li. This is an open-access article distributed under the terms of the Creative Commons Attribution License (CC BY). The use, distribution or reproduction in other forums is permitted, provided the original author(s) or licensor are credited and that the original publication in this journal is cited, in accordance with accepted academic practice. No use, distribution or reproduction is permitted which does not comply with these terms. 